\title{
Precision medicine and Mammalian Genome
}

\author{
Steve D. M. Brown ${ }^{1}$. Joseph H. Nadeau ${ }^{2}$ \\ Published online: 17 May 2019 \\ (c) Springer Science+Business Media, LLC, part of Springer Nature 2019
}

This year Mammalian Genome initiates a new focus on precision medicine and the role of mouse genetics and other model organisms in delivering new insights for genomic and precision medicine. Developments in precision and genomic medicine offer the promise of individualised and improved therapies for disease. However, the challenges of delivering precision medicine are formidable from cataloguing genetic variation in the human population, understanding the function of human genetic variation, eliciting disease mechanisms and ultimately translating this knowledge into improved treatment. There is a widespread recognition that model organisms, and in particular the mouse, will play a critical role in delivering biological knowledge that will underpin the genomic and precision medicine agenda. Studies in mouse genetics have the potential to unlock insights all the way from basic knowledge of gene function, through genetic networks and pathological mechanisms, to preclinical studies and the development of novel therapeutic strategies.

Given the importance of mouse genetics more generally to the precision medicine initiative, it is a key goal of Mammalian Genome over the coming year to highlight developments in mouse and mammalian genetics more broadly that exemplify and articulate the potential and excitement in this area. We have launched a series of reviews and commentaries over the coming year which cover many of the key achievements in mouse genetics and their impacts upon precision medicine, and which also look to future opportunities and challenges.

In this first issue, we publish four reviews. Two of the reviews (from Vashi and Justice and Tadenev and Burgess) discuss the importance and value of mouse models as preclinical models providing fundamental insights into disease mechanisms that enable utilisation of the models for the exploration of new therapeutic avenues. A recurring question is how much progress could be made in delivering novel treatments without new genetic models and the insights that they bring. New and more sophisticated models will be required and Leonard Shultz et al. discuss the challenges and opportunities that will arise from humanised mouse models for studies of immunological disease and their impact upon precision medicine. More widely, there is an unmet need for mouse models of human disease. Cacheiro, Haendel and Smedley from the International Mouse Phenotyping Consortium (IMPC) review the latest data from the IMPC cataloguing the very large number of novel models that are being generated. They discuss the integration of mouse and human phenomics data that will be critical for identifying key models for future pre-clinical studies as well as enhancing understanding of pathological and disease processes.

Finally, Mammalian Genome has revised its Aims and Scope to encourage the submission of research articles, reviews and commentaries that cover research in mammalian systems that impacts experimental aspects of interventions, therapeutics and precision medicine. We very much welcome reports of research on genetic, genomics, epigenetic and systems approaches that impact on the precision medicine initiative in its broadest sense. We look forward to your submissions be they research or review, and please do not hesitate to contact the Chief Editors to discuss your ideas and submissions. We look forward to hearing from you.

Publisher's Note Springer Nature remains neutral with regard to jurisdictional claims in published maps and institutional affiliations.
Steve D. M. Brown

s.brown@har.mrc.ac.uk

MRC Harwell Institute, Harwell, UK

2 Pacific Northwest Research Institute, Seattle, WA, USA 